\title{
Le règne de Louis XIV, ou la rupture définitive entre la société française et la monarchie
}

The Reign of Louis XIV: the ultimate split between French society and the monarchy

Jacques Bouveresse

\section{OpenEdition}

Journals

Édition électronique

URL : http://journals.openedition.org/add/332

DOI : $10.4000 /$ add. 332

ISSN : 2606-1988

\section{Éditeur}

Presses universitaires de Rouen et du Havre

\section{Édition imprimée}

Date de publication : 1 juin 2016

Pagination : 77-96

ISBN : 979-10-240-0599-7

ISSN : 1955-0855

\section{Référence électronique}

Jacques Bouveresse, "Le règne de Louis XIV, ou la rupture définitive entre la société française et la monarchie », Les Annales de droit [En ligne], 10 | 2016, mis en ligne le 08 janvier 2018, consulté le 18 décembre 2020. URL : http://journals.openedition.org/add/332 ; DOI : https://doi.org/10.4000/add. 332 


\title{
Le règne de Louis XIV, ou la rupture définitive entre la société française et la monarchie*
}

\author{
Jacques BOUVERESSE
}

Le long règne de Louis XIV, qui s'étend de 1661 à 1715, correspond dans sa première partie à l'apogée de la monarchie dite absolue. Précision de départ: il ne faut pas confondre absolutisme et despotisme. L'absolutisme, même s'il y a contradiction dans les termes, comporte des limites, des règles d'organisation et de fonctionnement, des institutions que le monarque doit respecter. Quant au despotisme, il désigne le gouvernement arbitraire, sans frein ni limites. L'absolutisme est la résultante d'une longue gestation historique. À mesure que le temps passe, la société française paraît de plus en plus soumise au roi. La noblesse, dès le $\mathrm{XVI}^{\mathrm{e}}$ siècle, est retenue à la cour dans une sorte de domesticité dorée. La cour, surtout celle de Versailles, fut le véritable antidote contre la turbulence et l'esprit frondeur des nobles. Grâce au concordat de Bologne de 1516, le roi a obtenu la disposition des principales dignités et bénéfices ecclésiastiques; il nomme les évêques et les abbés des principales abbayes, et s'assure ainsi la docilité du clergé. La bourgeoisie est tombée, elle aussi, dans l'orbite royale. Le système de la vénalité des charges et des offices la fixe irrésistiblement au service du roi. Artisans et commerçants sont encadrés par des groupements corporatifs étroitement soumis à l'autorité publique. Les paysans subissent les conséquences des pressions multiformes, politiques, économiques, fiscales, culturelles; sur eux, s'appesantit la tutelle de l'État, des centres urbains et des corps intermédiaires.

La soumission de la société au roi est réelle, les Français obéissent. Ils obéissent parce que leur culture, leur éducation les inclinent à l'obéissance: la France est l'héritière de Rome, patrie du droit, de

* Cet article reprend, en la modifiant, une conférence présentée à la faculté de droit de Rouen le 10 octobre 2014 à l'occasion d'une journée d'hommage à Pierre Le Pesant de Boisguilbert, figure éminente de l'histoire intellectuelle de la Normandie, et qui fut, dit-on, l'un des fondateurs de l'économie politique. 
la théorie de l'État, de la puissance publique. La France aussi est un pays catholique. Or, l'Église, modèle de société politique, est une structure monarchique dirigée par le pape; elle procède par affirmations dogmatiques, par voie hiérarchique, et refuse en matière religieuse le libre examen. Surtout, elle affirme que toute société, toute cité terrestre a besoin d'une autorité, d'un pouvoir. Le pouvoir est la conséquence du péché, de la chute originelle. Saint Paul et saint Pierre l'ont rappelé, «omni potestas a Deo ». L'Église enseigne donc le respect de l'autorité politique et la soumission au roi.

Mais la société aussi a des droits. L'homme chrétien sait qu'il est une image de Dieu, qu'il a une âme immortelle, et que s'il doit être un bon citoyen, un sujet obéissant, il ne doit pas tout à l'État. Ses droits fondamentaux ne viennent pas d'une concession faite par le pouvoir, il les tient du fait d'être un homme, de l'éminente dignité de la personne humaine. Les Français s'assignent des objectifs communs, plus ou moins consciemment : faire vivre, au cœur de la société, le principe d'égalité, proclamé par le christianisme. Dès le XIII ${ }^{\mathrm{e}}$ siècle, cette poussée égalitaire a obtenu des résultats: dans les villes, contre les seigneurs, les bourgeois ont arraché des chartes de franchise, le droit de gérer leurs affaires municipales; dans les campagnes, les serfs sont massivement affranchis et la plupart des paysans ont conquis, à la fin du Moyen Âge, la liberté personnelle. Plus tard, l'État offrira la protection de son droit et de son organisation aux propriétaires, aux épargnants, à l'ensemble de la classe moyenne en formation, en expansion autour de la bourgeoisie.

Rien de tout cela, certes, n'aurait été possible sans le roi. Mais l'absolutisme royal est seulement l'instrument de la volonté du peuple. La société n'obéit au roi que parce qu'elle commande. C'est elle qui fixe les objectifs : la constitution patiente, progressive, d'une immense classe moyenne qui n'est rien d'autre que le tiers état émancipé et prospère. L'absolutisme monarchique, en ce sens, n'est pas l'oppression de la société par le roi; il exprime la volonté sociale. C’est un mandat confié au roi, le moyen d'évincer les seigneurs qui exploitent les roturiers et de construire un État, une administration au service de la grande classe moyenne en formation dès le Moyen Âge, et qui prendra le pouvoir en 1789 .

Cette classe moyenne en expansion est aussi une espérance, la terre promise de tous les exclus, de tous les prolétariats. Ainsi, la volonté patiente d'élargir le groupe central des Français et, pour le tiers état, de s'emparer directement du pouvoir, vient de loin. Il n'y aurait jamais eu de 1789 si la France profonde, dès le Moyen Âge, ne s'était pas identifiée à ce grand projet de l'intégration sociale. Pour le réaliser, la société eut 
longtemps besoin du roi et lui confia un pouvoir absolu. Le roi a reçu un mandat: mettre au pas, par tous les moyens, les forces qui s'opposent à la réalisation de ce programme, la noblesse et une partie du haut clergé. Le pouvoir royal n'est absolu que dans la mesure où il s'attaque à la féodalité, aux féodalités. Pour le reste, il est limité par les prérogatives de la société, par des règles, des "privilèges » qui sont autant de signes de la vitalité sociale. Ce n'est pas de Dieu que le roi tient son pouvoir, mais de cette volonté décidée de la société. Ou plutôt, le Dieu qu'on invoque n'est rien d'autre que la volonté du peuple.

Ce que veut le peuple, c'est un État, une forte administration capable de concrétiser les objectifs de la société. Pour Tocqueville, auteur de L'Ancien Régime et la Révolution (1856), le tiers état est parvenu à subtiliser le gouvernement local, le pouvoir municipal, à la féodalité, mais il est trop faible encore, trop émietté pour exercer lui-même le pouvoir politique général. Il va donc, au Moyen Âge, le confier au roi, dénominateur de ses intérêts. Toute l'histoire de France est donc celle d'une continuité, de l'extension de l'État centralisé, de la mainmise de l'administration sur le corps social. Les conquêtes administratives des rois de France sont le trait dominant de notre histoire. Toutes les forces qui s'opposent à ce monopole sont anéanties. Et d'abord la noblesse, ou plus précisément, le principe aristocratique, donc l'idée même, la possibilité même de la légitimité d'une résistance à l'État. Le pouvoir central, devenu arbitraire, l'État, instrument de l'absolutisme, ne tolèrent plus les corps intermédiaires. Victoire du principe démocratique : car les sociétés démocratiques poussent au gouvernement centralisé; alors que les sociétés aristocratiques prônent la décentralisation, le gouvernement local. Au fond, Tocqueville voit dans l'existence d'une aristocratie, d'une élite au sens large, la garantie et le rempart de la liberté; alors que Guizot pense que l'aristocratie est un obstacle à la liberté. Mais pour les deux auteurs, l'histoire de France est bien celle de la croissance du pouvoir royal appuyé d'en bas sur le tiers état.

En définitive, le véritable bénéficiaire des théories absolutistes, ce n'est pas le roi mais l'État. La notion d'État se précise, à la fin $\mathrm{du} \mathrm{XvI}^{\mathrm{e}}$ et au début du XVII ${ }^{\mathrm{e}}$ siècle, en même temps que les doctrines absolutistes et chez les mêmes auteurs: Bodin, Coquille, Loyseau, Cardin Le Bret, Richelieu - et avec le sens que nous lui connaissons aujourd'hui. Pour ces auteurs, l'État présente trois caractères:

- il est d'abord autonome, c'est-à-dire doublement indépendant; indépendant des formes de gouvernement qu'il peut revêtir: monarchiques, aristocratiques, ou démocratiques ; indépendant aussi 
des hommes qui le gouvernent: ainsi en France, l'État n'appartient pas au roi qui en a simplement la responsabilité, son règne durant;

- ensuite, l'État est souverain: c'est à lui, et non pas au roi, que la souveraineté est attachée; le roi ne fait qu'exercer au nom de l'État, l'autorité souveraine;

- enfin, l'État est perpétuel: d'où procède l'idée d'une permanence de l'administration, d'une continuité des lois et des traités ; les rois s'en vont, tandis que l'État demeure.

À la fin de l'Ancien Régime, la féodalité a disparu depuis longtemps, l'État maintenant est fort, l'administration régulière et puissante. La société désormais peut se passer du roi et envisager de gouverner directement l'État par l'intermédiaire d'une élite bourgeoise. Le roi ne pouvait pas se passer de l'État, n'était rien sans lui; mais l'État peut se passer du roi. C'est ce qui arrivera sous la Révolution: la royauté disparaît, mais l'État va continuer, de plus belle et sans elle, à grandir et à fructifier ${ }^{1}$.

On n'en est pas là au début du règne de Louis XIV, qui correspond au moment le plus brillant de la monarchie française. Cependant, tout va se dégrader très vite à partir des années 1680. Ainsi que Paul Hazard l'a souligné, il y a longtemps déjà : les caractères de l'esprit du XVIII ${ }^{\mathrm{e}}$ siècle «se sont manifestés beaucoup plus tôt qu'on ne le croit d'ordinaire; on le trouve tout formé à l'époque où Louis XIV était encore dans sa force brillante et rayonnante; à peu près toutes les idées qui ont paru révolutionnaires vers 1760 , ou même vers 1789 , s'étaient exprimées déjà vers $1680^{2}$ ». Et, en plein XVIII ${ }^{\mathrm{e}}$ siècle, Diderot déclarait : «Nous avons eu des contemporains sous le règne de Louis XIV.» Thierry Maulnier a pu affirmer : «Le vrai siècle prométhéen de l'histoire des hommes n'est pas le $\mathrm{XVIII}^{\mathrm{e}}$ siècle, mais le XVII ${ }^{\mathrm{e}}$ siècle : le siècle où quelques hommes, parvenus à un degré d'audace, de résolution et de confiance dans l'exercice de la pensée qu'il faut bien dire superbe, osèrent prétendre, au nom de l'homme, à la victoire absolue sur le mystère universel et à l'empire sans limites de la pensée mathématique sur les forces de la nature ${ }^{3}$.» Le long règne du Roi-Soleil a donc été divisé en deux parties bien différentes: la première correspond à l'affirmation de l'absolutisme et d'un classicisme

1. Cette introduction reprend les considérations développées dans notre ouvrage: Histoire des institutions, de la vie politique et de la société françaises de 1789 à 1945, Rouen, PURH, 2012, p. 8-13.

2. Voir Paul Hazard, La crise de la conscience européenne (1680-1715), Paris, Boivin et $\mathrm{C}^{\text {ie }}, 1935$, t. I, «Introduction», p. IV.

3. Thierry Maulnier, "Introduction», dans Bernard de Fontenelle, Entretiens sur la pluralité des mondes habités, Paris, La Nouvelle France, 1945, p. 11. 
dans toute sa force; mais la seconde est marquée par le trouble des consciences, une mauvaise humeur presque générale et la diffusion d'un esprit de contestation qui, désormais, et jusqu’à la Révolution, ne cessera plus de se manifester.

\section{Le point culminant de la monarchie absolue : 1661-1685}

Dès 1661, quand il prend le pouvoir après la mort de son mentor, le cardinal Mazarin, Louis XIV s'impose d'emblée comme le maitre absolu. La Fronde, qui a troublé le pays quelques années auparavant, a démontré, s'il en était besoin, que les oligarchies nobiliaires et parlementaires, dès lors que le peuple était maintenu à l'écart, ne pouvaient remplacer la monarchie. Louis XIV gardait dans sa mémoire le souvenir des misères, des ruines et des massacres du milieu du siècle. Appuyé par le tiers état, il décida donc de gouverner par lui-même avec l'aide d'un petit nombre de collaborateurs compétents.

Son autorité est légitimée par les publicistes, les théoriciens de l'État dont les noms ont déjà été évoqués: Bodin, Coquille, Loyseau, Cardin Le Bret et Richelieu. Elle est soutenue aussi par les apologistes de l'absolutisme dont le plus célèbre est bien sûr Bossuet, évêque de Meaux et précepteur du fils aîné de Louis XIV, le Grand Dauphin. Bossuet donne à la doctrine absolutiste sa forme la plus achevée dans une sorte de cours destiné à son élève, et rédigé entre 1670 et 1679 : La politique tirée des propres paroles de l'Écriture sainte. La thèse de Bossuet repose sur l'idée que le roi reçoit son pouvoir directement de Dieu, sans l'intermédiaire du peuple. Dieu lui-même a choisi la dynastie qui gouverne la France, et ce choix est, au début de chaque règne, symbolisé par la cérémonie du sacre, en la basilique de Reims. De cette «théorie du droit divin » découle l'affirmation que la monarchie française est une monarchie pure de tout alliage, de tout mélange avec d'autres formes de gouvernement, aristocratiques ou populaires. Les sujets sont, en conséquence, privés de tout droit de contrôle envers le roi. Ils sont soumis au roi comme ils doivent l'être à Dieu, dont il est le lieutenant sur terre. L'obéissance au monarque est un devoir sacré.

Le roi va disposer d'une machine étatique déjà perfectionnée et dont il va améliorer le fonctionnement régulier. Ce système ignore la séparation des pouvoirs; et la confusion des pouvoirs entre les mains du roi signifie au fond que les pouvoirs, plutôt que de se limiter les uns les autres, sont associés et s'entraident en vue de faire triompher la souveraineté.

Le roi gouverne par conseil. Dès le $\mathrm{XII}^{\mathrm{e}}$ siècle, le recours au conseil est devenu une exigence de la pensée politique qui oblige le monarque. 
Le Conseil du roi est situé au sommet de l'appareil d'État. Identifié à la personne même du souverain, il est le centre nerveux du gouvernement. Gerson, chancelier de l'université de Paris, proclame ainsi dans un discours de 1405 (Vivat Rex): "Un roi sans un prudent conseil est comme la tête d'un corps sans yeux, sans oreilles et sans nez.» Louis XIV rappelle la nécessité pour le roi de "tout voir, tout écouter, tout connaître ». Il doit aussi, et surtout peut-être, s'entourer de conseils: «Délibérer à loisir, sur toutes les choses importantes, et en prendre de différentes gens, n'est pas, comme les sots se l'imaginent, un témoignage de faiblesse ou de dépendance, mais plutôt de prudence et de solidité. » Les mêmes recommandations se retrouvent dans ses Mémoires pour l'instruction $d u$ Dauphin où il affirme que «la discussion des matières se peut faire par eux [les conseillers], mais toutes les résolutions doivent être de vous [le futur roi] ». Au XVII ${ }^{\mathrm{e}}$ siècle, les conseils de gouvernement sont tous présidés par le roi. Le Conseil d'en haut règle les grandes affaires dans un cercle restreint de quelques personnes soigneusement choisies. Devant le Conseil des dépêches viennent les affaires administratives, les rapports et correspondances des intendants. Enfin, le Conseil royal des finances arrête la politique budgétaire et fiscale: montant de la taille, levée éventuelle de nouvelles taxes, équilibre du budget. Le principe du gouvernement par conseil est une originalité des institutions monarchiques. Le système est très souple puisque tout y dépend de la volonté du roi. Sous Louis XIV, la suprématie du Conseil du roi sur les officiers, les commissaires et les corps intermédiaires joue à plein. En revanche, le roi depuis les événements de la Fronde, se méfie des parlements et leur interdit de se parer du titre de «cours souveraines». En 1673, le roi ira même jusqu'à interdire aux parlements d'émettre des remontrances préalablement à l'enregistrement de ses ordonnances. C'est en vain que les parlements prétendent, et prétendront plus encore au XVIII ${ }^{\mathrm{e}}$ siècle, être le vrai Conseil du roi, le véritable " dépôt des lois ${ }^{4}$ ».

La centralisation se renforce inexorablement. Les provinces, les villes, après leur rattachement au royaume, s'efforcent de conserver une part d'autonomie, des assemblées et des privilèges particuliers. Cependant, les libertés locales s'affaiblissent. Les états provinciaux, rarement réunis, dominés par les agents du roi, n'offrent plus qu'un simulacre de liberté locale. Le roi prend l'habitude de nommer les maires des grandes villes,

4. Sur le Conseil du roi, voir Jacques Krynen, «La maîtrise royale du Conseil du roi », dans Histoires, peuples et droit. Mélanges offerts au professeur Jacques Bouveresse, Rouen, PURH, 2012, p. 89-98. Les citations de Gerson et de Louis XIV sont tirées de cet article (p. 92 et 97). 
ces échevins et ces consuls autrefois élus par les bourgeois du lieu. Les offices municipaux sont mis en vente. À la veille de la Révolution, les libertés locales, provinciales et urbaines, ont cédé presque partout sous le poids de la centralisation.

L'instrument le plus efficace de cette centralisation, c'est l'intendant, sorte de lien permanent et solide entre le pouvoir central et la réalité locale. L'intendant est un «commissaire » du roi : il est donc susceptible d'être révoqué ou déplacé sans garanties. Sur place, dans le cadre de sa "généralité », cet ancêtre du préfet napoléonien et des technocrates actuels, dispose de très larges pouvoirs.

Louis XIV entend également contrôler et surveiller les mouvements qui agitent la société. Il fera d'abord de la cour, comme on le sait, l'instrument d'abaissement de la noblesse. De cette noblesse française, ambitieuse, turbulente, travaillée par l'esprit d'intrigue et de trahison. Le roi imagina de la retenir et de la neutraliser dans une cour brillante où les fêtes, les bals, les réceptions se succédaient; où l'on se savait bien placé pour obtenir du maître des faveurs et de grands emplois. Le roi saura également faire de la guerre un ciment de l'unité nationale. La jeune noblesse, si ardente, sera enrôlée dans l'armée et les campagnes militaires, les camps, les sièges en fixeront la loyauté et stimuleront dans la nation entière la fierté du nom français.

Enfin, le roi s'emploie à surveiller les fluctuations de l'opinion publique. Cette opinion existait sans doute depuis toujours. Il y eut, d'ailleurs, des batailles d'opinion en plein Moyen Âge: ainsi Étienne Marcel luttant contre le dauphin Charles, révéla en 1358 la force de l'opinion parisienne. Cette opinion était pourtant trop versatile pour établir dans la durée une véritable emprise sur les événements. Elle ne pouvait se soutenir longtemps dans l'opposition, puisque le tiers état était groupé autour de son roi, de sa foi et de sa loi; rassemblé derrière un monarque, seul garant de l'ordre et mainteneur de la paix.

À la fin des guerres de Religion, l'opinion publique existe toujours, mais elle reste enfermée dans la gangue du système politique. Les Français ne conçoivent aucune alternative à la monarchie, il n'est pas question de la renverser; mais ils veulent un roi à leur goût: si Henri IV ne se convertit pas au catholicisme, il ne régnera jamais. Tandis que dans le nord de l'Europe, la religion du prince s'impose au peuple, en France, c'est la religion du peuple que le prince devra adopter. L'opinion ne va pas au-delà, elle ne remet pas en cause le principe même de la monarchie, d'une monarchie fidèle au mandat tacite que lui a confié la société. Un demi-siècle plus tard, la Fronde éclate: des centaines de pamphlétaires s'expriment; Paris est un immense foyer de bavardages, de racontars, 
d'intrigues, de complots. S'il faut à l'opinion un centre d'expression, ce centre existe bien; mais le principe directeur et fédérateur de l'opinion fait défaut : les Parisiens tournent autour du pouvoir royal sans parvenir à s'en détacher; d'où cette impression de flottement, de cacophonie. Ici encore l'esprit public, raisonnable et pondéré, l'emporte; car chacun sait, par-delà les débordements d'un instant, que personne, ni les parlements, ni la noblesse, ne peut exercer le pouvoir en France et maintenir l'ordre, sauf le roi.

Reste que la monarchie a bien senti le danger, en dépit du mépris affiché par la classe dirigeante pour l'opinion populaire. Cette opinion, il faut la canaliser, l'instrumentaliser, et, si le pouvoir manque son but, la réprimer. Louis XIV, en ce domaine, n'a négligé aucun détail. À partir de 1667 , un «lieutenant de Police » déploie sa vigilance et son adresse et La Reynie, titulaire de ce poste, est reçu longuement et chaque semaine par le roi. Le courrier des particuliers est ouvert par la poste aux lettres, ce qui permet à Louis XIV d'être informé des affaires particulières, même de moindre importance. La presse de l'Ancien Régime vit sous le régime de l'autorisation préalable et du privilège. Trois titres de journaux s'imposent et dominent à cette époque : la Gazette, le Journal des savants et le Mercure.

La France, dans cette première moitié du règne de Louis XIV, donne l'impression d'un bloc homogène, d'une société qui rêve d'accorder à chacun une place stable : aux paysans installés souvent depuis des siècles sur des tenures, dont ils finissent par se considérer comme les vrais propriétaires; aux artisans-commerçants, qui détiennent un monopole de leur métier dans le cadre des groupements corporatifs; aux officiers, propriétaires de leur charge, grâce au système de la vénalité des offices. Chacun est à sa place dans un ensemble dont la cohésion est assurée au sommet par la tutelle bienveillante du roi appuyé sur le tiers état.

Cet ensemble qui, de manière un peu abstraite, tend à une sorte d'harmonie et vise à offrir à chacun un port et un statut, correspond à l'idéal classique. Demeurer, éviter tout changement qui risquerait de détruire un équilibre miraculeux, c'est le souhait de l'âge classique. Elles sont dangereuses, les curiosités qui sollicitent une âme inquiète. Pascal déclare que tout le malheur des hommes vient d'une seule chose, qui est de ne pas savoir demeurer en repos dans une chambre. L'esprit classique, dans toute sa force, aime la stabilité; il voudrait être la stabilité même. La politique, la religion, la société, l'art, tout est soustrait aux discussions interminables, tout doit échapper à la critique insatisfaite. On voudrait, 
s'il était possible, arrêter le temps ${ }^{5}$. Ce n'est pas que l'esprit d'examen soit anéanti, il persiste chez les grands auteurs classiques, mais discipliné, amorti, puisqu'il s'agit de porter jusqu'à leur dernier point de perfection les chefs-d'œuvre qui exigent une longue patience. Cet esprit d'examen subsiste aussi chez les rebelles, qui attendent dans l'ombre le moment de mener la contestation générale. Ce moment ne va pas tarder à venir.

\section{Le grand renversement: 1685-1715}

Le décor ne change pas dans la deuxième moitié du règne de Louis XIV, mais l'opinion publique se modifie et le climat intellectuel se transforme profondément. À cet égard, deux dates significatives peuvent être retenues.

En 1685, Louis XIV révoque l'édit de Nantes, chassant ainsi hors de France près de 500 ooo protestants. Une élite intelligente et travailleuse doit s'exiler aux Pays-Bas, en Allemagne, en Angleterre; de cuisantes humiliations sont infligées à des milliers d'autres personnes. La révolte des «camisards» des Cévennes, au début de la guerre de Succession d'Espagne, est une première réplique. Durant trois ans, de 1701 à 1704, ces camisards réussirent à tenir la région et y établirent une "république ». L'opposition des protestants trouva de l'écho chez les jansénistes quand le roi, poussé par ses scrupules et par son souci de l'unité morale du pays, ferma Port-Royal des Champs (1709), le fit raser (1712), et pourchassa les évêques, les prêtres, les fidèles qui ne s'inclinaient pas devant la bulle Unigenitus, par laquelle le pape Clément XI condamnait solennellement, en 1713, le jansénisme. Dans ces conditions, une guerre religieuse commença, violente, impitoyable, haineuse. Rien ne rapproche davantage qu'une hostilité commune : protestants et jansénistes, sans devenir amis, se firent alliés. Se joignirent à eux une partie de la haute noblesse, écartée du premier cercle qui entourait le roi ; et surtout les parlementaires, qui en voulaient à Louis XIV de leur avoir interdit de présenter des remontrances préalables en 1673 , donc de se mêler de politique, en les réduisant à rendre la justice. Ces parlementaires gardaient aussi rancune au roi de s'être incliné devant Rome, car le gallicanisme, traditionnel dans ce corps, ne fléchissait pas. Enfin, ces hommes graves, s'ils ne pouvaient attaquer de front le pouvoir royal, s'en prenaient à la compagnie de Jésus et la poursuivaient de leurs mauvais procédés.

5. Paul Hazard, La crise de la conscience européenne, op. cit., t. I, «Introduction", p. III-IV. 
L'autre date importante est celle du début de la guerre dite de la ligue d'Augsbourg, en 1688. Cette guerre se terminera en 1697 sans avantage décisif. Lui succédera presque aussitôt l'interminable lutte autour de la Succession d'Espagne entre 1701 et 1713 . Ces conflits continuels devinrent vite impopulaires, car ils ruinaient le commerce, endeuillaient les familles, coûtaient très cher et entraînaient un alourdissement des impôts. L'économie stagnait et, même, régressait. Le royaume traversait une longue période de déflation. Les mauvaises récoltes et les famines de 1692 et surtout de 1709 engendrèrent la misère et stimulèrent les mécontentements.

Tout semblait maintenant tourner au désavantage du roi. $M^{\text {lle }}$ de Nantes, quatrième enfant de Louis XIV et de $\mathrm{M}^{\mathrm{me}}$ de Montespan, devenue $\mathrm{M}^{\mathrm{me}}$ la Duchesse par son mariage avec le petit-fils du Grand Condé, était de ceux qui versifiaient contre le roi :

Tant que vous fûtes libertin

Vous étiez maître du destin,

Landerirette.

Ah! pourquoi changer de parti?

Landeriri ${ }^{6}$.

Çà et là, des incidents éclataient, qui marquaient bien que les temps avaient changé. Les imprimeurs en profitaient. À Rouen, où le décret royal de 1704 fixait à dix-huit le nombre des imprimeurs, l'intendant dénonça la présence de trente et une imprimeries en 1706 ; les imprimeurs se débrouillaient pour élire des syndics conciliants qui les laissaient travailler à leur guise. Les gazettes, imprimées en Hollande, se diffusaient sans difficulté en France par la poste. La plus célèbre de ces feuilles, Les Nouvelles de la République des lettres, fut publiée à Amsterdam par Pierre Bayle de 1684 à 1687. Elle avait adopté la formule du Journal des savants.

Ainsi, les assaillants l'emportaient peu à peu. Quel contraste! Quel brusque passage! La hiérarchie, la discipline, l'ordre, que l'autorité monarchique se charge d'imposer, les dogmes qui règlent fermement la vie: telles étaient les règles qu'aimaient les hommes $\mathrm{du} \mathrm{XvII}{ }^{\mathrm{e}}$ siècle. À partir de la fin du règne de Louis XIV, les contraintes, l'autorité, les dogmes, voilà tout ce qui est, désormais, objet de détestation ${ }^{7}$. Le roi perd le contrôle de l'opinion publique et de la vie intellectuelle. La contestation s'élargit et se généralise également au domaine politique:

6. Citée par Mathurin-François-Adolphe de Lescure, Les philippiques de La GrangeChancel, Paris, Poulet-Malassis et de Broise, 1858, p. 43.

7. Paul Hazard, La crise de la conscience européenne, op. cit., t. I., «Introduction », p. i. 
il semble que le tiers état, soutenu par de nombreux publicistes, fasse silencieusement sécession.

La première offensive est menée par ce qu'il est convenu d'appeler les libertins. La pensée indépendante, qui, depuis Montaigne, n'a jamais abdiqué, se précise au début du $\mathrm{XVII}^{\mathrm{e}}$ siècle à la suite des guerres de Religion. Une cabale libertine, qui unit souvent à la liberté d'esprit une extrême liberté de mœurs, se forme dès 1615. Traqués par Richelieu, étroitement surveillés par Louis XIV, les libertins doivent se cacher pendant de longues années. Mais à partir de 1680, lorsque l'autorité royale se relâche, ils apparaissent au grand jour, affermissent leurs convictions et se préparent à la lutte. Le libertinage du $\mathrm{XVII}^{\mathrm{e}}$ siècle était une attitude d'esprit, l'autonomie morale de l'homme libre par opposition à l'homme soumis à l'autorité religieuse ${ }^{8}$. Le représentant le plus caractéristique de ce courant de pensée, fondé sur l'individualisme radical, est sans doute Saint-Évremond, déjà fort âgé, à la fin du $\mathrm{XVII}^{\mathrm{e}}$ siècle. Né en 1613 , mort en 1703 , Saint-Évremond traversa tout le Grand Siècle. Près de la moitié de sa vie s'écoula dans l'exil, en Angleterre la plupart du temps, et pour une courte période en Hollande. À Londres, il passait le meilleur de sa vie dans la maison d'Hortense Mancini, duchesse de Mazarin, l'une des nièces du cardinal. Il se créa de nouvelles habitudes, de nouvelles relations. Aussi, lorsqu'en 1689, Louis XIV lui fit connaitre qu'il pourrait rentrer en France, il refusa. Il continua de mener en Angleterre une existence agréable et demeura, jusqu'à ses derniers jours, épicurien et homme d'esprit ${ }^{9}$. L'indépendance de pensée de Saint-Évremond s'exprime surtout en morale et en littérature. En morale, il est un disciple fervent d'Épicure. Le souverain bien consiste pour lui à s'abandonner à la «bonne loi naturelle». Il s'arrangea pour passer doucement sa vie, plus ami de l'indolence que spectateur d'une vertu rigide. La sagesse ne nous a été donnée, suivant lui, que pour nous ménager des heures agréables. Le mot de vertu l'épouvante :

L'état de la vertu n'est pas sans peine. On y souffre une contestation éternelle de l'inclination et du devoir. Tantôt on reçoit ce qui choque, tantôt on s'oppose à ce qui plaît; sentant presque toujours de la gêne à faire ce que

8. Sur les libertins et le libertinage, voir Antoine Adam, Les libertins au xvir siècle, Paris, Buchet-Chastel, 1964, 323 p. ; Jacques Prévot, avec Thierry Bedouelle et Étienne Wolf (éd.), Écrivains libertins du XVII ${ }^{e}$ siècle, Paris, Gallimard, 1998, 1824 p. ; Michel Onfray, Contre-histoire de la philosophie, iii. Les libertins baroques, Paris, Grasset, 2008, $314 \mathrm{p}$.

9. Voir Charles-Antoine Gidel, «Étude sur la vie et les ouvrages de Saint-Évremond », dans Charles Hippeau (éd.), Euvres choisies de Saint-Évremond, Paris, Garnier frères, 1866, p. 1-70. 
l'on fait, et de la contrainte à s'abstenir de ce qu'on ne fait pas. Celui de la sagesse est doux et tranquille. La sagesse règne en paix sur nos mouvements et n'a qu'à bien gouverner des sujets, au lieu que la vertu avait à combattre des ennemis ${ }^{10}$.

Saint-Évremond est donc un partisan déterminé d'un individualisme assumé et radical.

Les progrès de l'esprit d'examen débouchent sur la contestation de la tradition et sur un scepticisme généralisé. La tradition, illustrée par la coutume, fut longtemps considérée comme l'expression respectable de l'histoire et de l'expérience accumulée par les générations successives. Le traditionalisme du $\mathrm{XVII}^{\mathrm{e}}$ siècle juge qu'une proposition est vraie si elle est admise par le consentement universel. Tel n'est pas l'avis de SaintÉvremond, de Bayle, de Fontenelle, qui présentèrent la tradition comme un amas de privilèges, de préjugés, de croyances absurdes, d'institutions inutiles ou nuisibles. La malice irréligieuse de Saint-Évremond réduit la foi, dans la Conversation du maréchal d'Hocquincourt avec le père Canaye, à une croyance sans fondements raisonnables ${ }^{11}$. En 1684, dans son court traité De l'origine des fables, Fontenelle ${ }^{12}$ attribue la croyance au surnaturel à l'ignorance des premiers hommes qui imaginèrent, pour expliquer les phénomènes naturels, l'intervention de divinités supérieures. À travers l'exemple des mythes païens, c'est, bien entendu, le christianisme qui est visé. En 1687, l'auteur rouennais prolonge sa réflexion dans son Histoire des oracles. Les premiers chrétiens, nous dit-il, ont cru que les oracles païens étaient l'œuvre des démons; Fontenelle entend démontrer que les oracles ne pouvaient être rendus par les démons puisqu'ils étaient dus aux artifices des prêtres qui

10. Ibid., p. 65 .

11. Parmi les éditions relativement récentes des œuvres de Saint-Évremond qui nous intéressent ici, on mentionnera : Euvres en prose, Paris, Didier, 1962 ; Lettres, Paris, Didier, 1967; Écrits philosophiques, Paris, Alive, 1996. Et, sur Saint-Évremond lui-même: Albert-Marie Schmidt, Saint-Évremond ou l'humaniste impur, Paris, Cavalier, 1932; Henry Thomas Barnwell, Les idées morales et critiques de SaintÉvremond: essai d'analyse explicative, Paris, PUF, 1957, 234 p.; Claude Taittinger, Saint-Évremond ou le bon usage des plaisirs, Paris, Perrin, 1990, $227 \mathrm{p}$.

12. Bernard Le Bovier de Fontenelle est né le 11 février 1657 à Rouen. Sa mère était la sœur de Pierre et Thomas Corneille. Il mourut centenaire en 1757. Les œuvres complètes de Fontenelle ont fait l'objet d'une réédition récente: CEuvres complètes de Fontenelle, Paris, Fayard, 9 vol., 1990-2001 (édition d'Alain Niederst). Voir également la Revue Fontenelle (dix numéros édités de 2003 à 2013 par les Presses universitaires de Rouen et du Havre). Sur le philosophe lui-même, parmi de très nombreux ouvrages, on peut citer le livre récent de François Bott, L'entremetteur, esquisses pour un portrait de M. de Fontenelle, Paris, PUF, 1991, $111 \mathrm{p}$. 
exploitaient la crédulité des fidèles. "Avec ses livres sur les oracles et sur les fables, il a écrit une histoire de tous les mensonges. Il a dépeint cette terrible crédulité humaine qui, venant de notre misère et de notre faiblesse, nous fait admettre si facilement ce qui répond à nos espérances ${ }^{13}$. " Enfin, dans ses Entretiens sur la pluralité des mondes de 1686, Fontenelle met l'astronomie à la portée du grand public cultivé. Pour vulgariser le système de Copernic, il est amené à réfléchir sur quelques idées: scepticisme à l'égard de la métaphysique et du merveilleux, satire des hommes qui se croient au centre de l'univers et affirmation du relativisme. On trouve cette maxime dans ses Entretiens: «Il faut ne donner que la moitié de son esprit aux choses que l'on croit, et en réserver une autre moitié libre, ou le contraire puisse être admis s'il en est besoin ${ }^{14}$.»

La déférence quelque peu ironique avec laquelle Fontenelle s'incline à l'avance devant la majesté encore imposante du dogme est de style libertin. "Les philosophes du XVIII ${ }^{\mathrm{e}}$ siècle, écrit Thierry Maulnier, auront plus d'audace et de violence, mais cette désinvolture dans le respect, ces phrases de soumission écrites sur des pages où il est aisé de discerner les filigranes de l'insolence, tout cela ne peut nous tromper: Fontenelle est déjà de leur camp ${ }^{15}$.» La vocation de Fontenelle était précisément de ménager les premières entrevues entre le vieux $\mathrm{XVII}^{\mathrm{e}}$ siècle et le jeune XVIII ${ }^{\mathrm{e}}$.

Pierre Bayle est également d'un scepticisme radical en philosophie, comme le montre son Dictionnaire historique et critique, recueil d'articles concernant des noms propres historiques ou géographiques ${ }^{16}$. De

13. François Bott, L'entremetteur..., op. cit., p. 21.

14. Cité par François Bott, ibid., p. 86-87.

15. Ibid., p. 24.

16. Pierre Bayle est né en 1647 dans un village des Pyrénées. Son père était pasteur de l'Église réformée. Tour à tour précepteur et professeur, Pierre Bayle mena une vie errante. Il séjourna ainsi et successivement à Toulouse, Genève, Rouen, Paris, Sedan, puis s'installa définitivement à Rotterdam. La révocation de l'édit de Nantes en 1685 marqua une étape décisive dans la maturation de sa pensée. Il critiqua la politique de Louis XIV et réfléchit à l'importance de la tolérance dans une société. Bayle mourut en 1706, dix ans après la sortie de son Dictionnaire qui eut un immense succès tout au long du XviII ${ }^{e}$ siècle. Voir Pensées diverses écrites à un docteur de Sorbonne à l'occasion de la comète qui parut au mois de décembre 1680, 1682 (édition récente: Paris, Flammarion, "GF », 2007, sous le titre: Pensées diverses sur la comète); Dictionnaire historique et critique, 1697 (édition moderne: Dijon, Les Presses du réel, 2013). Sur l'œuvre de Bayle, voir: Paul Hazard, La crise de la conscience européenne, op. cit., t. I, chap. v, «Pierre Bayle», p. 131-154; Élisabeth Labrousse, Pierre Bayle, La Haye, Nijhoff, 2 vol., 1963 et 1964; Élisabeth Labrousse, Notes sur Bayle, Paris, Vrin, 1967 ; Gianluca Mori, Bayle philosophe, Paris, Champion, 
cette énorme compilation se dégage l'impression que l'histoire humaine regorge de crimes. D'où le scepticisme à peu près total de Bayle. La contradiction perpétuelle des témoignages, l'incertitude même qui pèse sur les faits historiques aboutissent à ruiner l'histoire et donnent l'impression que les opinions opposées sont également incertaines; cette incertitude des connaissances et des interprétations est le fondement de la tolérance. Par-là, Bayle est le père des philosophes du XviII ${ }^{\mathrm{e}}$ siècle, qui puiseront sans cesse dans son Dictionnaire. Voltaire est imprégné de son œuvre, et l'Encyclopédie est inspirée par la méthode et par l'esprit de son Dictionnaire.

Le scepticisme ébranle la tradition et la religion; en revanche, il respecte l'expérience scientifique et a tendance à tout examiner à la lumière de la raison pour en tirer des conclusions pratiques. Telle était déjà l'attitude des libertins vers le milieu du $\mathrm{XvII}^{\mathrm{e}}$ siècle. Tel est aussi le souci de Fontenelle qui, dans ses Entretiens manifeste sa foi dans la méthode scientifique ${ }^{17}$ et affirme sa croyance au progrès qui fera de l'homme le maître de la nature ${ }^{18}$. Le propos de Pierre Bayle est du même ordre. Dans ses Pensées sur la comète (1682), il affirme la primauté de l'expérience et de l'esprit scientifique, l'incompatibilité entre le mystère religieux et la raison.

Libertinage, poussée de l'individualisme et de l'esprit d'examen, scepticisme déterminé, foi dans la raison humaine, dans le développement de la science et du progrès : ainsi, à peu près toutes les attitudes mentales dont l'ensemble aboutira à la Révolution française ont été adoptées avant la fin du règne de Louis XIV. Passage de la stabilité au mouvement. Dès

2001; Antony McKenna et Gianni Paganini (dir.), Pierre Bayle dans la République des lettres: philosophie, religion, critique, Paris, Champion, 2004; Hubert Bost, Pierre Bayle, Paris, Fayard, 2006; Bayle historien critique et moraliste, Turnhout, Brepols, 2006 ; Gianni Paganini, Le débat des modernes sur le scepticisme. Montaigne, Le Vayer, Campanella, Hobbes, Descartes, Bayle, Paris, Vrin, 2008.

17. Il y a, chez Fontenelle, une conviction très forte: rien n'est certain or ce qui est démontré par la science. S'il est une vérité, elle ne peut être que scientifique. Voir Willy de Spens, «Fontenelle ou l'indifférent", introduction à l'Histoire des oracles, Paris, Union générale d'éditions, 1965, p. VI-VII.

18. Cette croyance dans le progrès débouche chez Fontenelle sur une forme d'optimisme rationnel: «Un bon esprit cultivé est, pour ainsi dire, composé de tous les esprits des siècles précédents, ce n'est qu'un même esprit qui s'est cultivé pendant tout ce temps-là... Il est maintenant dans l'âge de virilité, où il raisonne avec plus de force et a plus de lumières que jamais. " Voir Martin Stern, "Jean-Jacques, que me-veux-tu? Rousseau critique de Fontenelle», Revue Fontenelle, $\mathrm{n}^{\mathrm{o}}{ }_{5}, 2007$, p. 88-89. 
que le classicisme a cessé d'être un effort, une volonté, une adhésion réfléchie, pour se transformer en habitudes et en contraintes, les tendances contestataires et novatrices, toutes prêtes, ont repris leur force et leur élan. Les discussions se tendent et s'aigrissent; et les autorités perdent progressivement le contrôle de la vie intellectuelle. Il y a, cependant, plus grave encore: le contrat tacite entre le roi et son peuple, qui faisait du monarque le rempart et le défenseur du tiers état, ce contrat a perdu sa valeur fondamentale. Le roi, pas plus qu'il ne contrôle l'opinion publique et la vie intellectuelle, ne peut désormais empêcher la vie politique de se déployer en dehors de son emprise.

Les auteurs les plus pénétrants soulignent d'abord que le tiers état, souvent repoussé à la marge des interprétations politiques, est bien au cœur de la vie sociale. Vauban montre par exemple l'importance déterminante du tiers état et surtout des couches populaires:

C'est encore la partie basse du peuple, écrit-il, qui, par son travail et son commerce, et par ce qu'elle paie au roi, l'enrichit et tout son royaume; c'est elle qui fournit tous les soldats et matelots de ses armées de terre et de mer, et grand nombre d'officiers, tous les marchands et les petits officiers de judicature; c'est elle qui remplit tous les arts et métiers; c'est elle qui fait tout le commerce et les manufactures de ce royaume, qui fournit tous les laboureurs, vignerons et manouvriers de la campagne; qui garde et nourrit les bestiaux; qui sème les blés et les recueille; qui façonne les vignes et fait le vin; et pour achever de le dire en peu de mots, c'est elle qui fait tous les gros et menus ouvrages de la campagne et des villes. Voilà en quoi consiste cette partie du peuple, si utile et si méprisée, qui a tant souffert, et qui souffre tant à l'heure que j'écris ceci ${ }^{19}$.

Cet extrait capital est tiré de l'ouvrage majeur de Vauban, le Projet d’une dîme royale, écrite en 1699 et imprimée en 1707, l'année de la mort de son auteur.

Or le tiers état est mal gouverné. Des réformes sont donc indispensables. Les préconisations sont ici multiples. Fénelon, l'auteur des Aventures de Télémaque (1699), se retranche derrière l'éloquence sacrée pour marquer son hostilité profonde à Louis XIV et à son absolutisme :

Avez-vous cherché les gens les plus désintéressés et les plus propres à vous contredire? Avez-vous pris soin de faire parler les hommes les moins empressés à vous plaire, les plus désintéressés dans leur conduite, les plus capables de condamner vos passions et vos sentiments injustes? Quand vous avez trouvé des flatteurs, les avez-vous écartés? Vous en êtes-vous défié? Non, non, vous n'avez point fait ce que font ceux qui aiment la vérité, et qui

19. Sébastien Le Prestre de Vauban, Projet d'une dîme royale..., s. 1., 1707, p. 17. 
méritent de la connaître. Pendant que vous aviez au dehors tant d'ennemis qui menaçaient votre royaume encore mal établi, vous ne songiez au-dedans de votre nouvelle ville qu'à y faire des ouvrages magnifiques... Vous avez épuisé vos richesses; vous n'avez songé ni à augmenter votre peuple ni à cultiver les terres fertiles... Une vaine ambition vous a poussé jusques au bord du précipice. À force de vouloir paraître grand, vous avez pensé ruiner votre véritable grandeur ${ }^{20} \ldots$

L'attaque est violente. Fénelon sera bientôt disgracié et « exilé » dans son archevêché de Cambrai.

Néanmoins, les réformes préconisées par Fénelon ne vont pas dans le sens de l'émancipation du tiers état. On voit bien, à lire son texte, que le peuple est ici l'objet de la sollicitude du prélat, et non pas le sujet actif et l'artisan de son propre destin. Fénelon, en effet, prépare avec ses amis de la haute noblesse, les ducs de Chevreuse, de Beauvilliers et de Saint-Simon un plan de gouvernement, les Tables de Chaulnes (1711), comportant la restauration des gouverneurs, des états généraux et provinciaux dotés de pouvoirs législatifs et administratifs, dominés par la noblesse. Ce projet qu'on peut, sans hésitation qualifier de « rétrograde", comporte aussi la suppression des intendants. La révolution des ducs et pairs préconisée par Fénelon annonce toute une série de projets d'inspiration aristocratique, qui vont proliférer au XVIII ${ }^{\mathrm{e}}$ siècle.

Les vues de Vauban et de Boisguilbert sont d'une portée bien différente. Vauban voudrait réhabiliter le «menu peuple», le soulager par une répartition plus équitable des impôts, sans exemption ni privilège: la « dîme royale ", payée par tous, porterait aussi bien sur les revenus de toutes les professions (salaires, industries, immeubles) que sur les produits de la terre. Une dîme établie sans arbitraire coûterait moins et rendrait plus. Boisguilbert, lieutenant-général au bailliage de Rouen a publié en 1697 un ouvrage d'un esprit tout nouveau, qui étudie l'origine de la richesse et de la prospérité économique, Le Détail de la France, sous-titré La cause de la diminution de ses biens et la facilité du remède. L'appauvrissement de la France résulte, selon l'auteur, de l'abandon de la culture et de la sous-consommation, idée qui sera reprise par les physiocrates. Boisguilbert montre que la France, jadis le plus riche royaume du monde, a perdu une part importante de ses revenus annuels; et ce déficit augmente sans cesse. La taille est si injustement répartie qu'elle pèse sur les pauvres en épargnant les riches; les pauvres sont devenus misérables, le royaume tout entier court vers sa perte. Il suggère en

20. François de Fénelon, Les Aventures de Télémaque, fils d'Ulysse..., LeydeAmsterdam, Wetstein-Chatelain, 1761, p. 167, livre XII. 
conséquence la suppression des douanes intérieures et, pour la première fois, la répartition de l'impôt selon les revenus ${ }^{21}$.

Boisguilbert et Vauban, loin d'être des révoltés, cherchent à assainir les finances et à procurer au roi les ressources qu'il recherche désespérément. Ils n'en agissent pas moins en intrus qui empiètent sur un domaine, autrefois réservé : le Projet d'une dîme royale sera condamné au feu. Mais il est remarquable qu'à la fin du siècle, sous un régime absolu, des écrivains aient voulu rappeler le roi à ses devoirs et lui suggérer des réformes. L'esprit français s'oriente vers d'autres conceptions du gouvernement.

Le roi se trouvait également dépossédé par une affirmation étonnante, résumée en une formule dans les éditions $\mathrm{du} \mathrm{xIX}^{\mathrm{e}}$ siècle: "Les lois de l'ordre économique ne se violent jamais impunément ${ }^{22}$." Le roi aura beau faire, il ne peut, tout absolu que soit son pouvoir, violer impunément les lois économiques. On est ici, dès la fin du XviI ${ }^{\mathrm{e}}$ siècle, au commencement de cette science économique qui se déploiera une cinquantaine d'années plus tard.

Le roi est encore dépossédé par la pensée politique d'origine anglaise. Louis XIV était le représentant glorieux du droit divin. Par une théorie toute différente de celle de Bossuet, Hobbes soutenait de même la nécessité du pouvoir absolu, le Léviathan. Le droit naturel va naître d'une philosophie qui nie le surnaturel, le divin, et substitue l'ordre immanent de la nature à l'action et à la volonté personnelle de Dieu. John Locke, en politique, a combattu la théorie du droit divin dans son livre de 1689: Deux traités de gouvernement. Selon lui, en droit naturel, les hommes sont libres et égaux; c'est en vertu d'un pacte social qu'ils délèguent le pouvoir à une autorité pour qu'elle protège cette liberté et cette égalité primitives. Le gouvernement doit donc être élu et, à la mode anglaise, garantir la liberté du peuple par la séparation des pouvoirs.

21. Sur Boisguilbert, on peut consulter: Jacqueline Hecht, Pierre de Boisguilbert ou la naissance de l'économie politique, Paris, INED, 1966 ; Bertrand Tremblay, La conceptualisation de l'activité économique selon Boisguilbert, thèse de l'université de Montréal, 1973; Pierre Rosanvallon, "Boisguilbert et la genèse de l'État moderne », Esprit, janvier 1982, p. 32-52; Gilbert Faccarello, Aux origines de l'économie politique libérale: Pierre de Boisguilbert, Paris, Anthropos, 1986, 312 p.; Félix Cadet, Pierre de Boisguilbert précurseur des économistes 1646-1714, Paris, Guillaumin, 1871 (réédition Institut Coppet, 2014); Albert Talbot, Les théories de Boisguilbert et leur place dans l'histoire des doctrines économiques, New York, Franklin, 1971 (réédition Institut Coppet, 2014).

22. Par exemple dans le volume Économistes-financiers $d u$ XvIII siècle [Vauban, Boisguilbert, Jean Law, Melon, Dutot], Eugène Daire (éd.), Paris, Gullaumin, 1843, résumé du chap. vi du Détail de la France, p. 218. 
De manière générale, le principe d'autorité est remis en cause. On insistait, dans l'ancienne France, sur les devoirs de l'homme envers les communautés auxquelles il appartient: la communauté humaine, d'où le respect de ses devoirs envers Dieu; la communauté familiale, d'où l'obéissance due par la femme à son mari et par les enfants à leurs parents; la communauté politique, d'où le devoir d'obéissance au roi, lieutenant de Dieu sur terre. À l'énumération des devoirs, la philosophie nouvelle va préférer l'énoncé des droits de l'être humain: droits de la conscience individuelle, droits de la raison, droits de la critique, droits de l'homme et du citoyen ${ }^{23}$. Ces droits sont universels et imprescriptibles. Quant à l'autorité qui règle arbitrairement les rapports des sujets et du prince, elle doit être rejetée et remplacée par un droit nouveau, d'où sortira peut-être le bonheur, un droit politique qui fixe les rapports sociaux avec l'idée que ce sont les peuples eux-mêmes qui dirigent leur propre destin.

En dépit des interdictions et des censures, l'effervescence intellectuelle semble irrépressible. Les vieilles théories du droit divin sont submergées par des représentations politiques nouvelles qui préfigurent, par leur audace, les grands débats de la deuxième moitié du XviII ${ }^{\mathrm{e}}$ siècle. Paradoxalement, l'essentiel n'est peut-être pas là ; écrivains et publicistes ne lancent des écrits de portée significative que dans la mesure où ils portent la parole et les aspirations du tiers état. Or, la société française, dans ses dernières années du règne de Louis XIV, est silencieuse. Le temps des barricades, des soulèvements, des émotions populaires est terminé depuis longtemps. La société n'est pas pour autant subjuguée; son silence est lourd de réprobation. Il n'est pas excessif de prétendre que le mandat tacite qui liait la monarchie et le tiers état depuis deux siècles ne vaut plus. Les contemporains n'en avaient peut-être pas parfaitement conscience. Selon la fameuse formule de Marx, «les hommes ne savent pas l'histoire qu'ils font ». Les historiens, ici, ont un avantage: avec un recul de trois siècles, il leur est plus facile de démêler l'écheveau des événements et de leur signification. On devine mieux aujourd'hui que la confiance perdue entre le roi et son peuple ne se rétablira pas, et que la Révolution est en marche. Le tiers état a trop souffert au cours des trente années qui finissent le règne: la stagnation économique, la misère, suite des mauvaises récoltes, des guerres et des accidents climatiques, la lourdeur des impôts, l'isolement du roi, tout a contribué à détourner les Français d'un monarque lointain, et qui paraît indifférent. Le roi est enfermé

23. Paul Hazard, op. cit., t. I, «Introduction», p. IV-v. 
à Versailles, entouré d'une petite cour de favoris, d'une oligarchie de privilégiés, coupés des réalités sociales. Les gens subissent en silence, avec patience, les mauvais procédés des autorités. Mais leur confiance dans leur roi est ébranlée, elle est même perdue et ne reviendra plus. Et c'est sous les huées de la foule des badauds que le corbillard du roi s'acheminera de Versailles à la nécropole de Saint-Denis.

Tout est donc fini en 1715. Le roi, pourtant, continue à jouer imperturbablement son rôle sur le théâtre d'ombres qu'est devenu son palais. Avec une montre, dit Saint-Simon, on peut savoir à toute heure du jour quelle est l'occupation et l'emploi du temps du roi. La machine monarchique fonctionne sans à-coups : le jeu alterne avec la promenade dans les jardins, les réunions avec les ministres suivent la messe et la chasse précède souvent le souper. Dans l'accomplissement de sa tâche, Louis XIV fait preuve d'un véritable héroïsme; ainsi, trois semaines avant sa mort, debout pendant deux heures dans la galerie des Glaces, il reçoit l'ambassadeur de Perse, alors que sa jambe est déjà attaquée par la gangrène. S'il s'applique, c'est qu'il semble croire à la durée de son œuvre. Rien ou presque ne nous autorise à croire que, pour le roi, à l'heure de disparaître, la digue dressée au prix de tant d'efforts ne tiendra pas mieux que le château fort des enfants anéanti par la marée du soir. Il y a aussi ces paroles prêtées par la tradition au roi moribond : «Je m’en vais, mais l'État subsistera toujours".

Il est vrai que l'État s'est structuré et affermi au cours de ce long règne, que l'appareil administratif, sans être, et de loin, aussi perfectionné qu'aujourd'hui, a poussé ses tentacules jusqu'aux extrémités du corps social. Mais si l'État se renforce, la monarchie, elle, est gravement délégitimée, frappée d'une maladie de langueur qui la conduira à la mort. Cette situation nouvelle, le roi ne paraît pas en avoir mesuré la gravité. Il n'est pas possible de dire que Louis XIV eût pu reprendre à son compte cette déclaration de Pompée, rapportée par Dion Cassius : «Tu te souviens de ces serpents qui, quand nous arrivâmes en Épire, effaçaient derrière nous la trace de nos pas? D'autres serpents viendront, et tout sera effacé ${ }^{24}$.»

Professeur émérite d'histoire du droit

Faculté de droit, université de Rouen

24. Citée par Henry de Montherlant, Va jouer avec cette poussière. Carnets 1958-1964, Paris, Gallimard, 1966, p. 200. 